\title{
The Relationship between Type 2 Diabetes Mellitus and Risk of Prostate Cancer: Literature Review and Critical Appraisal
}

\section{Timothy Shun Man Chu*}

Newcastle University, UK

"Corresponding author: Timothy Shun Man Chu, Newcastle University, UK, Tel: +44 (0) 746338 6460; E-mail: T.Chu1@newcastle.ac.uk

Received date: February 09, 2018; Accepted date: March 12, 2018; Published date: March 16, 2018

Copyright: ( 2018 Timothy SM Chu. This is an open-access article distributed under the terms of the Creative Commons Attribution License, which permits unrestricted use, distribution, and reproduction in any medium, provided the original author and source are credited.

\begin{abstract}
Background: Type 2 Diabetes Mellitus and Prostate Cancer are two common illnesses. However, there was not extensive research done to determine the relationship between the two diseases.

Objective: This article aims to investigate the relationship between Type 2 Diabetes Mellitus and the risk of Prostate Cancer, and some possible underlying mechanisms.

Method: Several papers published within 5 years in this area are critically appraised and reviewed, including meta-analyses with large scale cohorts.

Results: Type 2 Diabetes Mellitus is linked to a moderately decreased risk of Prostate Cancer, as seen in recent evidences. Possible underlying mechanisms include changes in hormonal profiles, genetics, and use of diabetic drugs (Metformin).

Conclusion: While Type 2 Diabetes Mellitus might be protective against Prostate Cancer risk, more evidence as to how confounding variables such as ethnicity, lifestyle, and co-morbidities modify the overall risk is needed, as related information in this area is scarce.
\end{abstract}

Keywords: Diabetes mellitus; Prostate cancer; Hormonal profiles; Metformin; Prostate cancer risk; Type 2 diabetes

\section{Introduction}

Type 2 Diabetes Mellitus (T2DM) is a metabolic disease characterised by chronic hyperglycaemia. It occurs when the pancreas fails to secrete adequate insulin or when body develops insulin resistance [1]. The prevalence of Diabetes in the UK was estimated at 4.5 million in 2016, and around $90 \%$ of which suffers from T2DM. Males are at a higher risk of developing T2DM as audits have suggested that of all adult diabetics, 56\% are men [2]. Other risks of T2DM include obesity, genetic predisposition, and age.

There is a long list of complications associated with T2DM, such as diabetic retinopathy and neuropathy. Moreover, T2DM has been found to be associated with higher incidences of several types of cancer, including pancreatic, liver, and kidney cancer [3]. On the other hand, around 1 in 7 men will get diagnosed with Prostate Cancer (PCa) in his lifetime [4]. PCa is also a public health concern as it is the most common cancer in men in the UK with over 46,000 new cases diagnosed annually [4].

The aim of this review is to investigate the relationship between T2DM and PCa, and its significance to diabetic patients. This is done by reviewing and critically appraising several papers published within 5 years in this area. This review will then address the possible underlying mechanisms and reasons of the linkage between T2DM and PCa.

\section{Literature Review}

\section{Relationship between T2DM and PCa}

As mentioned above, emerging studies suggests that men with T2DM are associated with an increased risk of several cancers. However, recent epidemiologic evidence on PCa risk is conflicting, and is presented below:

The European Prospective Investigation into Cancer and Nutrition (EPIC) was a study involving more than 500,000 voluntarily recruited participants from 10 European countries. Tsilidis et al. [5] conducted a cohort study on the participants of EPIC. In the study, 139,131 men were followed up for an average of 12 years to trace for incidences of $\mathrm{PCa}$. This is done by collecting and analysing standardized questionnaires regarding personal health status, lifestyle and nutrition from the participants. Multivariable hazard ratios (HR) and 95\% confidence intervals (CI) were estimated using cox proportional hazards models, and were adjusted for confounding PCa risk factors such as BMI and smoking status to minimalize bias. On the other hand, Bansal et al. [6] undertook a meta-analysis of observational studies (29 cohort and 16 case-control studies) retrieved from a comprehensive literature search using PubMed. A total of 8.1 million participants and $132,331 \mathrm{PCa}$ cases were involved. The primary measure was the pooled risk ratio (RR) of PCa. It was calculated using the random-effects model to account for the heterogeneity among the studies. As the research methodology employed was meta-analysis, which lies at a higher level in the hierarchy of evidence [7], a higher reliability than Tsilidis' cohort study is therefore expected. 
Both studies aim to examine the association between T2DM and the risk of PCa. Tsilidis et al. found that of all PCa cases $(n=4,531)$, a selfreported history of DM is associated with a $27 \%$ reduced risk of prostate cancer [5] (HR, 0.73; 95\% CI, 0.62-0.85). Similarly, Bansal et al. recorded a $14 \%$ reduction of PCa risk (RR, 0.86; 95\% CI, 0.80-0.92) associated with T2DM patients [6] $(\mathrm{n}=921,582)$. Although the percentage reduction in PCa risk found in the two studies varies by more than $10 \%$, their overall results are consistent with other major research and genetic studies in this field $[8,9]$. Moreover, Tsilidis et al. discovered that men with increasing duration of DM ( $>12$ years) have an even lower PCa risk, when compared to non-diabetic men (HR, 0.64; $95 \%$ CI, 0.51-1.48). Both studies show no connection between T2DM and severity grade of PCa.

Tsilidis et al. assessed other variables such as socio-demographics and lifestyle characteristics in addition to diabetes, and adjusted statistical models for them with the hope to minimalize their effects on $\mathrm{DM}$ and/ or PCa risk. This strengthens the reliability of results and therefore increases the validity of the paper. However, their large sample size $(\mathrm{n}=139,131)$ was unequally distributed between men with (3.7\%) and without (96.3\%) history of DM diagnosis, reducing the validity of results. Also, in their study, men with DM were on average older, less physically active, and had a higher BMI than those without. This bias could lead to an underestimation of the reduction of PCa risk as these are all known or suspected PCa risk factors [10], hence decreasing the accuracy of the results.

Bansal's research includes articles before and after the advent of prostate specific antigen (PSA). This means that the study might have a detection bias, as the implementation of PSA testing might have changed the scope of disease that was diagnosed as PCa [6]. Bansal et al. tried to combat this possible bias by running a subgroup analysis on men diagnosed before and after the widespread usage of PSA testing. They proved a non-significant variation of results between pre-PSA $(\mathrm{RR}=0.87)$ and PSA testing era $(R R=0.90)$, hence increasing the validity of the results. Also, this study's huge participating population ( $\mathrm{n}>8.1$ million) and inclusion of large scale cohorts $(\mathrm{n}>30,000)$ reduced the observational and recall bias and strengthened the study generalizability. In general, the results of Bansal's study are statistically significant as they used only cohort studies reporting RRs, and casecontrol studies reporting odds ratios, with $95 \%$ CIs respectively [6].

Both Tsilidis et al. and Bansal et al. failed to differentiate between T1DM and T2DM patients in their studies, reducing the accuracy of the results as T1DM and T2DM have different metabolic profiles and etiology [11] that could potentially affect the risks of PCa in different ways. Tsilidis et al. were aware of this flaw and subsequently performed a sensitivity analysis that excluded men with possible T1DM, which showed that this has minimal effect on the PCa risk [5] (HR, 0.74; 95\% CI, 0.62-0.87).

To conclude, the two studies showed an inverse relationship between T2DM and the risk of PCa. Both studies have identified a link to clinical practice, that is DM may increase the risk for an enlarged prostate and therefore lead to a decreased likelihood to detect cancer with biopsy [12].

\section{Possible Underlying Mechanisms}

\section{Hormonal profiles}

A different hormonal profile in T2DM patients has been hypothesized to be a cause for the reduced PCa risk in this group of patients. These hormones include insulin, insulin-like growth factor-I (IGF-1), insulin-like growth factor binding protein-III (IGFBP-3), and sex hormones including testosterone ( $\mathrm{T})$ and sex hormone binding globulin (SHBG). A decreased insulin and IGF-1 could inhibit prostate carcinogenesis because they are positively related with the growth of both normal and cancerous prostate cells [13], and are reportedly risk factors of $\mathrm{PCa}$ [14]. A decreased T and SHBG may have protective effects too, as $\mathrm{T}$ undergoes conversion to an active metabolite that binds androgen receptors in prostate cells, which then increases transcription and proliferation of both normal and cancerous cells [15].

In Tsilidis' study mentioned in the previous section, a secondary aim was to explore the mechanisms through which DM might influence PCa risk, by comparing the circulating concentrations of the above hormones in men with and without DM [4]. 30mL blood samples were taken from a total of 626 men $(\mathrm{DM}=37$, non-DM control $=589$ ) and hormonal profiles were measured (for IGF-1 they used $78 \mathrm{DM}$ and 1,449 non-DM controls). A comparable prospective cohort study was done by Kasper et al. [16] with a similar purpose. The study involved 3172 men $(D M=171$, non-DM control $=3,001)$ from the Health Professionals Follow-up Study (HPFS), in which investigations like those completed in EPIC were done on 51,529 U.S. health professionals. Blood samples were collected from the study population and the same hormones were measured. Statistical analyses were performed in Tsilidis' and Kasper's studies. Both used a linear regression model controlling for other variables such as laboratory batch and BMI to minimalize bias of results due to these factors. In Kasper's research, the diabetics were further divided into four groups based on their years since diagnosis to explore how hormonal levels might change in accordance with DM timeline.

Both studies found decreased plasma levels of all the above hormones in men with diabetes compared with those without (Table 1). On top of that, Kasper et al. found that over time, C-peptide (marker of insulin secretion), IGF-1, IGFBP-3 levels and ratio of T to SHBG (showing bioavailable $\mathrm{T}$ ) decrease whilst $\mathrm{T}$ and SHBG levels increase in DM patients [16].

The general strengths and limitations of Tsilidis' study were discussed in the previous section. An additional limitation of their study is their small sample size was unevenly distributed among "DM" ( $\mathrm{n}=37)$ and "Non-DM" ( $\mathrm{n}=589)$, which damages their result generalisability and validity. In Kasper's study, due care was taken to lower the degree of bias and ensure credibility. Firstly, they conducted studies confirming minimal degradation of samples during processing, storing and shipping thus reducing possible detection bias. Secondly, the specimens were blinded to avoid any exposure of information at the laboratory, with masking QC samples added to the batches to reduce bias and monitor precision of the measurements. Thirdly, they tried to validate the self-reporting DM cases and confirmed $94 \%$ of the initial reports, lessening the recall bias from the study population.

However, both studies failed to address the possible effects of DM medications on hormonal levels in T2DM patients. This reduces the validity and reliability of results as evidence has shown that common anti-diabetic drugs such as Metformin might pose influences on hormonal levels $[17,18]$, thus affecting the risk of PCa (to be discussed in later sections in this report). This is also an important clinical link for doctors to consider. When managing T2DM patients presenting with other co-morbidities that might be affected by a change in hormonal levels, medications should be prescribed with caution. 
Overall, studies in this field have detected changes in the hormonal profiles of T2DM patients that could attribute to a reduced risk of PCa among these patients. Although recent articles have fortified this link [19], more work is needed for the understanding of hormonal interactions with specific receptors in T2DM patients that contribute to the decreased PCa risk.

\begin{tabular}{|c|c|c|c|c|}
\hline & \multicolumn{2}{|l|}{ Tsilidis et al. } & \multicolumn{2}{|c|}{ Kasper et al. } \\
\hline & $\begin{array}{l}\text { Non-DM } \\
(n=589, \text { for } \\
\text { IGF-1 } \\
n=1,449)\end{array}$ & $\begin{array}{ll}\ln _{n=78} & (n=37, \\
\text { for } & \text { IGF-1 }\end{array}$ & $\begin{array}{l}\text { Non-DM } \\
(n=3,001)\end{array}$ & $\mathrm{DM}(\mathrm{n}=171)$ \\
\hline Hormones & \multicolumn{2}{|l|}{ In $\mathrm{nmol} / \mathrm{L}$} & \multicolumn{2}{|c|}{$\begin{array}{l}\text { In } \mathrm{ng} / \mathrm{mL} \text {, in } \mathrm{nmol} / \mathrm{L} \text { for } \\
\text { SHBG }\end{array}$} \\
\hline C-peptide & - & - & 2.41 & 2.82 \\
\hline Total Testosterone & 15.9 & 14.1 & 4.82 & 4.13 \\
\hline SHBG & 43.3 & 39.6 & 74.3 & 69.3 \\
\hline IGF-1 & 19.8 & 19.4 & 184.5 & 180.9 \\
\hline IGFBP-3 & 130 & 117 & $3,603.70$ & $3,521.90$ \\
\hline
\end{tabular}

Table 1: Hormonal Profiles by DM-Status $[4,16]$.

\section{Genetics}

It is evident that genetics is a risk factor for both T2DM [20] and PCa [21]. Common genetic variations in both diseases might offer an explanation as to how T2DM is negatively related to the risk of PCa. Recent genome-wide association (GWA) studies suggested that variants in some genes could affect both PCa and T2DM risk [22,23]. Two genetic studies were reviewed in this section to summarise the findings in this field.

Pierce et al. [24] identified 18 single nucleotide polymorphisms (SNPs) that have been confirmed as T2DM risk variants from a literature review. They then generated risk scores (risk allele count and genetic relative risk) for these SNPs for T2DM, for PSA-screened PCa cases $(\mathrm{n}=1,171)$ and matched controls $(\mathrm{n}=1,101)$ from the Cancer Genetic Markers of Susceptibility study (CGMS). A statistical analysis using logistic regression was then performed to estimate odds ratios (OR) and 95\% CIs for the association between the risk scores and PCa risk. Meyer et al. [25] also selected 13 SNPs that are related to T2DM and genotyped them. Based on 397 PCa cases among 6,642 men in the Atherosclerosis Risk in Communities Study (ARIC), these SNPs were tested for association with $\mathrm{PCa}$ using cox proportional hazard regression model. Crude HRs and 95\% CIs were then calculated. Both studies intended to examine the association between T2DM risk alleles and PCa risk. Pierce et al. focused more on the collective effects of these genes while Meyer et al. focused on the effects individual SNPs.

Both studies found an inverse relationship between some of the T2DM-susceptible SNPs and the risk of PCa, their findings are summarized in Table 2 (only statistically significant associations shown). Pierce et al. also found that a higher number of T2DM risk allele count ( $>20$ risk alleles) yields a further reduction in PCa risk (OR, 0.77; 95\% CI, 0.60-0.99), compared with a lower number of T2DM risk allele count (17-18 risk alleles. OR, 0.99; 95\% CI, $0.77-1.25)$. Contrary to the above findings, results from Meyer's study showed that one SNP (CAPN10 rs3792267 G allele) is positively linked to $\mathrm{PCa}(\mathrm{HR}, 1.20 ; 95 \% \mathrm{CI}, 1.00-1.44)$. This could be explained by the fact that CAPN10 involves in neoplastic processes, by coding for serine proteases that play a role in cell cycle and cell migration [26].

\begin{tabular}{|c|c|c|c|c|c|c|}
\hline \multirow[b]{2}{*}{$\begin{array}{l}\text { Studies } \\
\text { Reviewed }\end{array}$} & \multirow[t]{2}{*}{ Gene } & \multirow[t]{2}{*}{ SNP } & \multirow{2}{*}{$\begin{array}{l}\text { Risk } \\
\text { Allele }\end{array}$} & \multicolumn{3}{|c|}{ Factor Adjusted } \\
\hline & & & & OR/ HR & $95 \% \mathrm{Cl}$ & p value \\
\hline \multirow{2}{*}{$\begin{array}{l}\text { Pierce et al. } \\
\text { (recording } \\
\text { OR) }\end{array}$} & FTO & rs 8050136 & A & 0.87 & $0.77-0.98$ & 0.02 \\
\hline & HNF1B & rs4430796 & G & 0.87 & $0.77-0.97$ & 0.02 \\
\hline \multirow{4}{*}{$\begin{array}{l}\text { Meyer et al. } \\
\text { (recorded } \\
\text { HR) }\end{array}$} & CAPN10 & rs 3792267 & G & 1.20 & $0.88-1.44$ & $<0.1$ \\
\hline & SLC2A2 & rs5400 & G & 0.85 & $0.71-1.01$ & $<0.1$ \\
\hline & TCF7L2 & rs7903146 & T & 0.84 & $0.68-1.03$ & - \\
\hline & UCP2 & rs660339 & C & 0.83 & $0.72-0.96$ & $<0.05$ \\
\hline
\end{tabular}

Table 2: Association between T2DM risk variants and PCa risk [24,25].

As the two genetic studies were focusing on the genes of T2DM, rather than T2DM status itself, their observations would less likely be due to a reverse causation or confounding factors such as lifestyle. Hence, we would expect a higher validity of the results from these studies. Additionally, Pierce's research has a higher accuracy and reliability as their sample size for PCa cases was higher (1,171 vs. 397$)$.

Although both were case-control studies, their different methodologies mean they have different strengths and limitations. Pierce's study involved a case group and a matched control group with similar numbers. During the selection process, they removed firstdegree relatives in the study population, ensured participants had at least one official PCa screening prior to enrolment and screened them for the duration of the study [24]. These actions minimised selection, recall and performance bias respectively and enhanced research validity. However, Meyer et al. did not mention any work done to reduce recall bias as their inclusion of cases rely mainly on questionnaires and telephone interviews [25], without much official verification. Both studies may have limited credibility for the results of some genes as they either had substantial missing data for some SNPs (Pierce's study), or low power in detecting moderate effects of SNPs ( $<80 \%$, self-identified by Meyer et al.).

In summary, the two articles reviewed support an inverse association between the T2DM-susceptible genes and PCa risk. Future studies should focus more on individual SNPs to establish the genetic mechanisms of such association. Research in this area also show that T2DM patients and their families might benefit from genetic counselling, as they could understand more about their risks for DM, $\mathrm{PCa}$, and/or other diseases.

\section{Anti-Diabetic Drugs (Metformin)}

As explained in previous sections, drug treatments in T2DM might have a role in the overall reduced PCa risk. Effects of Metformin specifically are discussed in this review as it is used extensively in the management of T2DM. Metformin was thought to possess anti-cancer properties, since it was repeatedly associated with a decreased risk of various types of cancer [27]. While exact mechanisms behind this remain unclear, some hypothesized that it is linked to Metformin's inhibitory effects on cancer cell proliferation pathways [27]. Haring et al. [28] and Häggström et al. [29] independently conducted nationwide cohort studies to evaluate the risk of PCa among users of Metformin. 
Haring's study was retrospective and involved 78,615 men that were randomized to the regular PSA screening arm $(n=31,866)$ or the control standard care arm $(\mathrm{n}=48,278)$. They first confirmed an inverse relationship between T2DM and PCa risk by comparing anti-diabetic drug users $(n=15,578)$ to non-users $(n=63,037)$. Specific effects of Metformin were then investigated through comparing the PCa risk between users of Metformin and other oral anti-diabetic drugs [28]. Due to the retrospective nature of the study, confounding covariates that may have influenced the results could not be controlled. Nevertheless, Haring et al. tried to reduce interference of these covariates by doing subgroup statistical analyses keeping other variables constant.

Häggström's study was prospective and consisted of 612,846 men. Similar procedures to confirm protective effect of T2DM on PCa risk were completed. Effects of Metformin were examined in a subgroup study of T2DM men $(n=25,238)$ with mean follow-up time of 4 years, by comparing PCa risk of Metformin users to those not treated with anti-diabetic drugs [29]. The relatively short follow-up time of this research means potential $\mathrm{PCa}$ cases might not be recognised, thus causing detection bias.

The two studies show conflicting results (Table 3). Haring et al. found that among anti-diabetic drug users, Metformin decreased overall PCa risk (HR, 0.81; 95\% CI, 0.69-0.95) in a dose-dependent manner [28] while Häggström et al. found no association between Metformin and PCa risk in T2DM patients [29].

\begin{tabular}{|l|l|l|l|l|}
\hline Studies reviewed & $\begin{array}{l}\text { Number of } \\
\text { Diabetics }\end{array}$ & & $\begin{array}{l}\text { Number of PCa } \\
\text { cases }\end{array}$ & \multicolumn{2}{|l|}{ Factor Adjusted } \\
\cline { 4 - 5 } & & HR & $\mathbf{9 5 \%} \mathbf{~ C l}$ \\
\hline Haring et al. & 8,989 & 762 & 0.81 & $0.69-0.95$ \\
\hline Häggström et al. & 3,691 & 157 & 0.96 & $0.77-1.19$ \\
\hline
\end{tabular}

Table 3: PCa risk in Metformin users [28,29].

In Haring's study, the decrease in risk was significant only in the screening arm, suggesting a major flaw that PSA screening might have modified the association between Metformin and PCa risk. This is supported by the fact that baseline PSA was lower in Metformin users in the study. As PCa is commonly diagnosed based on biopsies performed for elevated PSA, lower baseline PSA could lead to fewer diagnostic biopsies performed and PCa detected [28]. Thus, the recorded risk reduction might be caused by detection bias, which lowers results reliability and validity. Häggström et al. combatted this bias by using data on Benign Prostatic Hyperplasia as a proxy for diagnostic activities, increasing their research validity. Furthermore, the results of Haring's study might not be generalized and reproduced in other ethnic groups and countries as the cohort consisted of Caucasian men in Finland only, despite of their relatively large sample size.

A weakness in Häggström's study was a lack of data on risk factors of $\mathrm{PCa}$, resulting in potential residual confounding. However, other forms of bias were kept to a minimal in their research. For example, they counteracted a possible detection bias, that is the effect of duration of $\mathrm{T} 2 \mathrm{DM}$ on $\mathrm{PCa}$ risk, by dividing participants into subgroups according to time since $\mathrm{T} 2 \mathrm{DM}$ diagnosis.

Both studies fail to separate entirely the effects of Metformin from the potentially masking effects of underlying diabetes on PCa risk, hence lowering their results accuracy and validity. Both authors realized this issue, and attempted to reduce bias. Haring et al. addressed this by analysing the specific effects of Metformin using other anti-diabetic drugs as reference [28]. Conversely, Häggström et al. struggled to solve the problem by investigating a subgroup of men with onset of T2DM after the commencement of the research [29]. However, they fail to present any relevant findings.

In closing, effect of Metformin on PCa risk in T2DM patients is disputable. More work is needed in this field as current evidence is conflicting and insufficient to synthesize a conclusion, although few recent papers have shown that metformin lowers risk and/or mortality of PCa $[30,31]$.

\section{Conclusion}

T2DM is undoubtedly linked to a moderately decreased risk of PCa as seen in recent evidence $[5,6,8,9]$, and the strength of this report lies in inclusion of a meta-analysis with large scale cohorts. Several proposed mechanisms behind this link are the hormonal changes in T2DM patients, genetic association between the risks of the two diseases, and the influences of anti-diabetic drugs.

Whether this association is due the above suggested mechanisms or the lower PSA levels in T2DM leading to a lower detection rate of PCa in these patients [32] needs to be confirmed in future higher power studies. It is because the link between T2DM and PCa risk seem to be less apparent before PSA-testing is introduced and in studies that adopt other means to detect PCa [8]. This is also a limitation of this report as most articles reviewed contain this detection bias.

While T2DM might be protective against PCa risk, more evidence as to how confounding variables such as ethnicity, lifestyle, and comorbidities modify the overall risk is needed, as related information in this area is scarce.

\section{References}

1. Barnett A (2012) Type 2 diabetes. (1st edn), Oxford University Press, Oxford.

2. Diabetes UK (2016) Facts and Stats [Internet] p. 2-6.

3. Giovannucci E, Harlan D, Archer M, Bergenstal R, Gapstur S, et al. (2010) Diabetes and Cancer: A Consensus Report. CA Cancer J Clin 60: 207-221.

4. Prostate cancer statistics (2017) Cancer Research UK.

5. Tsilidis K, Allen N, Appleby P, Rohrmann S, Nöthlings U, et al. (2014) Diabetes mellitus and risk of prostate cancer in the European Prospective Investigation into Cancer and Nutrition. Int J Cancer 136: 372-381.

6. Bansal D, Bhansali A, Kapil G, Undela K, Tiwari P (2013) Type 2 diabetes and risk of prostate cancer: a meta-analysis of observational studies. Prostate Cancer Prostatic Dis 16: 151-158.

7. Greenhalgh T (2014) How to read a paper: The Basics of Evidence-based Medicine. (1stedn), BMJ Publishing Group Limited, Chichester.

8. Kasper J, Giovannucci E (2006) A Meta-analysis of Diabetes Mellitus and the Risk of Prostate Cancer. Cancer Epidemiol Biomarkers Prev 15: 2056-2062.

9. Gudmundsson J, Sulem P, Steinthorsdottir V, Bergthorsson J, Thorleifsson G, et al. (2007) Two variants on chromosome 17 confer prostate cancer risk, and the one in TCF2 protects against type 2 diabetes. Nat Genet 39: 977-983.

10. Torti D, Matheson G (2004) Exercise and Prostate Cancer. Sports Medicine 34: 363-369.

11. Ozougwu O (2013) The pathogenesis and pathophysiology of type 1 and type 2 diabetes mellitus. J Physiol Pathophysiol 4: 46-57. 
Citation: Timothy SM Chu (2018) The Relationship between Type 2 Diabetes Mellitus and Risk of Prostate Cancer: Literature Review and Critical Appraisal. J Diabetes Metab 9: 787. doi:10.4172/2155-6156.1000787

Page 5 of 5

12. Sarma AV, Parsons JK, McVary K, Wei JT (2009) Diabetes and benign prostatic hyperplasia/lower urinary tract symptoms-what do we know? Urol 182: S32-S37.

13. Djiogue S, Nwabo Kamdje A, Vecchio L, Kipanyula M, Farahna M, et al. (2012) Insulin resistance and cancer: the role of insulin and IGFs. Endocr Relat Cancer 20: R1-R17.

14. Roddam A (2008) Insulin-like Growth Factors, Their Binding Proteins, and Prostate Cancer Risk: Analysis of Individual Patient Data from 12 Prospective Studies. Ann Intern Med 149: 461-471.

15. Coffey D, Isaacs J (1979) Prostate cancer: A series of workshops on the biology of human cancer. Report no. 9 (UICC technical report series). (1stedn), International Union Against Cancer, Geneva.

16. Kasper J, Liu Y, Pollak M, Rifai N, Giovannucci E (2008) Hormonal profile of diabetic men and the potential link to prostate cancer. Cancer Causes Control 19: 703-710.

17. Campagnoli C, Berrino F, Venturelli E, Abbà C, Biglia N, et al. (2013) Metformin Decreases Circulating Androgen and Estrogen Levels in Nondiabetic Women With Breast Cancer. Clin Breast Cancer 13: 433-438.

18. Ozata M, Oktenli C, Bingol N, Ozdemir I (2001) The Effects of Metformin and Diet on Plasma Testosterone and Leptin Levels in Obese Men. Obes Res 9: 662-667.

19. Yanase T, Kawanami T, Tanaka T, Tanabe M, Nomiyama T (2017) Impact of metabolic disorders on prostate cancer growth: Androgen and insulin resistance perspectives. Reprod Med Biol 16: 252-257.

20. Kato N (2013) Insights into the genetic basis of type 2 diabetes. J Diabetes Investig 4: 233-244.

21. Prostate cancer (2017) Genetics Home Reference.

22. Gudmundsson J, Sulem P, Steinthorsdottir V, Bergthorsson J, Thorleifsson G, et al. (2007) Two variants on chromosome 17 confer prostate cancer risk, and the one in TCF2 protects against type 2 diabetes. Nat Genet 39: 977-983.

23. Frayling T, Colhoun H, Florez J (2008) A genetic link between type 2 diabetes and prostate cancer. Diabetologia 51: 1757-1760.

24. Pierce B, Ahsan H (2010) Genetic Susceptibility to Type 2 Diabetes Is Associated with Reduced Prostate Cancer Risk. Hum Hered 69: 193-201.

25. Meyer T, Boerwinkle E, Morrison A, Volcik K, Sanderson M, et al. (2010) Diabetes Genes and Prostate Cancer in the Atherosclerosis Risk in Communities Study. Cancer Epidemiol Biomarkers Prev 19: 558-565.

26. Carragher NO, Frame M (2002) Calpain: a role in cell transformation and migration. Int J Biochem Cell Biol 34: 1539-1543.

27. Kasznicki J, Sliwinska A, Drzewoski J (2014) Metformin in cancer prevention and therapy. Ann Transl Med 2: 57

28. Haring A, Murtola T, Talala K, Taari K, Tammela T, et al. (2017) Antidiabetic drug use and prostate cancer risk in the Finnish Randomized Study of Screening for Prostate Cancer. Scand J Urol 51: 5-12.

29. Häggström C, Van Hemelrijck M, Zethelius B, Robinson D, Grundmark B, et al. (2016) Prospective study of Type 2 diabetes mellitus, anti-diabetic drugs and risk of prostate cancer. Int J Cancer 140: 611-617.

30. Zingales V, Distefano A, Raffaele M, Zanghi A, Barbagallo I, et al. (2017) Metformin: A Bridge between Diabetes and Prostate Cancer. Front Oncol $7: 243$.

31. Hankinson S, Fam M, Patel N (2017) A review for clinicians: Prostate cancer and the antineoplastic properties of metformin. Urol Oncol 35: 21-29.

32. Pierce B (2012) Why are diabetics at reduced risk for prostate cancer? A review of the epidemiologic evidence. Urol Oncol 30: 735-743. 